\title{
Histologic recovery among children with celiac disease on a gluten-free diet. A long-term follow-up single- center experience
}

\author{
Oana Belei ${ }^{1}$, Andreea Dobrescu², Rodica Heredea ${ }^{3}$, Emil Radu lacob $^{4}$, Vlad David ${ }^{4}$, Otilia Marginean ${ }^{1}$
}

\author{
${ }^{1}$ First Pediatric Clinic, "Victor Babes" University of Medicine and Pharmacy, Timisoara, \\ Romania \\ 2Department of Genetics, "Victor Babes" University of Medicine and Pharmacy, \\ Timisoara, Romania \\ ${ }^{3}$ Department of Pathology, “Victor Babes” University of Medicine and Pharmacy, \\ Timisoara, Romania \\ 4Department of Pediatric Surgery, "Victor Babes" University of Medicine and \\ Pharmacy, Timisoara, Romania
}

Submitted: 30 May 2017

Accepted: 14 July 2017

Arch Med Sci 2018; 14, 1: 94-100

DOI: https://doi.org/10.5114/aoms.2018.72241

Copyright $\odot 2017$ Termedia \& Banach

\section{Abstract}

Introduction: Celiac disease (CD) is defined by gluten-induced immune-mediated enteropathy, affecting approximately $1 \%$ of the genetically predisposed population. The immunologic response to gluten causes characteristic intestinal alterations with gradual development. Histologic recovery of intestinal architecture was reported to occur within 6-12 months after starting a gluten-free diet, simultaneously with clinical remission. The aim of this study was to assess the rate and timing of histologic recovery among children with CD on a gluten-free diet, diagnosed and followed in an academic referral pediatric center during a 10 -year period.

Material and methods: 105 biopsy-confirmed CD children underwent follow-up small intestinal biopsies within at least 1 year after dietary gluten withdrawal. Further biopsies were performed if villous alterations were persistent. The Marsh classification modified by Oberhuber was used to score the histologic injuries.

Results: In all 19 cases with Marsh type II at diagnosis, villous alterations normalized to Marsh type 0 within the first year. From 86 children enrolled with Marsh type III lesions, histologic remission was observed in $81.4 \%$ after 1 year, $91.8 \%$ within $2-3$ years and $97.6 \%$ in long-term follow up ( $\geq 3$ years). Two (2.3\%) patients with concomitant selective IgA deficiency had symptoms of malabsorption and persisting villous atrophy lasting more than 3 years despite a gluten-free diet. There was a significant statistic difference between the proportion of children with Marsh type IIIA, type IIIB and Marsh type IIIC respectively that achieved histologic recovery within 1 to 2 years after gluten withdrawal. There were more children with partial $25(92.6 \%)$ and subtotal villous atrophy 30 (88.2\%) showing histologic improvement, compared to only $15(60 \%)$ patients with total villous atrophy that recovered within the first 2 years of diet ( $p=0.01$ and $p=0.02$ respectively).

Conclusions: Histologic recovery in $C D$ after starting a gluten-free diet in children takes at least 1 year and might be incomplete only in a small proportion of children, mainly associated with IgA immunodeficiency. Systematic follow-up of children with CD and persistent malabsorption syndrome is needed in order to avoid secondary complications.

Key words: endoscopy, intestinal biopsy, villous atrophy.

\author{
Corresponding author: \\ Emil Radu lacob \\ Department of Pediatric \\ Surgery \\ "Victor Babes" University \\ of Medicine and Pharmacy \\ E. Murgu Square no. 2 \\ 300041 Timisoara, Romania \\ Phone: +40 745614590 \\ E-mail: radueiacob@umft.ro
}




\section{Introduction}

Celiac disease (CD) is defined as an immune-mediated enteropathy caused by intolerance to gluten in genetically susceptible individuals associated with human leukocyte antigen (HLA) DQ2 or DQ8, affecting approximately $1 \%$ of the population [1]. Celiac disease incidence has increased over the decades, due to increased exposure to gluten, awareness of the broad spectrum of clinical presentation and the availability of sensitive and specific diagnostic tools [2]. The spectrum of clinical presentation is heterogeneous, ranging from typical gastrointestinal symptoms to extra-intestinal manifestations, mild laboratory abnormalities or even asymptomatic patients detected by active screening among risk groups. Serum antibody assays are nowadays widely used for CD screening, but the final diagnosis requires typical findings in small-bowel biopsy. The standard diagnosis tool for gluten enteropathy is intestinal biopsy showing characteristic villous lesions with a few exceptions. According to European Society for Pediatric Gastroenterology, Hepatology and Nutrition (ESPGHAN) revised criteria, intestinal biopsy can be omitted in selected circumstances, based on a combination of symptoms, antibodies and HLA, including symptomatic patients with immunoglobulin A (IgA) anti-tissue-transglutaminase (tTG) levels above 10 times the manufacturer's cut-off and positive endomysial antibodies (EMA) [3].

The immunologic response to gluten in celiac patients causes microscopic alterations of the small intestinal mucosa, including increased lymphocyte density in villous epithelium, crypt hyperplasia and villous atrophy $[4,5]$. In CD, the characteristic gluten-induced small intestinal mucosal injury develops gradually $[4,6]$. The spectrum of mucosal changes contains two separate measurable parameters: inflammation reflected by intraepithelial lymphocytic infiltration, and morphological damage which includes villous atrophy and crypt hyperplasia [7]. Histologic recovery of small intestinal mucosa is assumed to occur within 6 to 12 months after starting a gluten-free diet, simultaneously with clinical remission [8]. The wellknown classifications for histological assessment of intestinal alterations among patients with CD are the one described by Marsh and modified by Oberhuber and another produced by Corazza and Villanacci $[4,9,10]$ These are the most practical histological scores used in daily work. Not only negative serology and symptom-free status are important in follow-up of celiac patients, but also a morphologically healed mucosa is a prerequisite for the long-term well-being of a patient [11]. Surprisingly, follow-up studies on small intestinal histology recovery in CD children are scarce.
The aim of this study was to assess the rate and timing of histologic recovery among children with CD on a gluten-free diet, diagnosed and followed in an academic referral pediatric center during a 10-year period.

\section{Material and methods}

The authors conducted a 10-year prospective follow-up study at an academic referral pediatric center from the Western part of Romania. We enrolled 105 children with CD (68 girls, 37 boys; mean age: $4.6 \pm 1.2$ years, range: $1-18$ years) who were diagnosed and followed in our hospital between January 2007 and March 2017. Inclusion criteria were: children with typical intestinal symptoms (chronic diarrhea, abdominal distension and failure to thrive) or atypical symptoms and extra-digestive manifestations (chronic constipation, recurrent abdominal pain, non-specific increased level of liver enzymes, iron deficiency anemia resistant to oral iron therapy, short stature/growth failure, osteoporosis, amenorrhea), positive serology at diagnosis and characteristic initial histological findings corresponding to at least Marsh type II or more severe injuries classified as Marsh type IIIA, type IIIB and type IIIC, children adherent to a strict gluten-free diet, with negative IgA and/or IgG tTG serology at follow-up.

Exclusion criteria were: children diagnosed with CD according to ESPGHAN revised criteria, in whom the initial intestinal biopsy was omitted, celiac children presenting intestinal alterations classified as Marsh type I at diagnosis - only intraepithelial lymphocyte infiltration, without crypt hyperplasia or villous atrophy, children non-compliant with gluten-free diet with persistence of symptoms and positive serology at follow-up and children with underlying diseases accompanied by possible non-celiac villous injuries such as giardiasis, Crohn's disease, autoimmune enteropathy, eosinophilic enteritis, cow's milk protein allergy, intestinal lymphangiectasia, Whipple disease or amyloidosis.

IgA deficiency is estimated to affect $2 \%$ to $3 \%$ of patients with $C D$, representing an increase of 10 to 15 fold over the general population [12]. Hence, IgA deficiency was not an excluding criteria and CD serology was IgG based at diagnosis and during follow-up in children with selective IgA deficiency. CD histological confirmation and follow-up had the same protocol as for IgA sufficient celiac children in this study.

All legal guardians/parents of patients enrolled in this study signed written informed consent prior to inclusion. The study was approved by the Ethical Committee of the host institution. The work was conducted in compliance with the Local Institutional Review Board for Human Sub- 
jects Research Committee requirements, including the Declaration of Helsinki - Ethical Principles for Medical Research Involving Human Subjects.

After written informed consent was obtained, follow-up small intestinal biopsies were performed within 1 to 2 years after starting a gluten-free diet in all enrolled children. Further biopsies were taken only in case of persistent villous atrophy at the control biopsy or in case of symptoms relapsing. The authors divided follow-up small intestinal biopsies in time frames: to 2 years, 2 to 3 years and 3 years or more (long-term follow-up).

The follow-up small intestinal biopsies were taken by upper digestive endoscopy from the second part of the duodenum, obtaining at least four samples in each case. Histological interpretation of the intestinal samples was performed by an experienced pathologist using the Marsh classification modified by Oberhuber [9]. This scoring system comprises a spectrum of intestinal mucosal injuries as follows: Marsh type 0 is described as normal mucosal pattern, Marsh type I as infiltrative lymphocytic enteritis (normal mucosal architecture with lymphocytes infiltration in villous epithelium - more than 30 lymphocytes per 100 enterocytes), Marsh type II hyperplastic (lymphocytic enteritis with crypt hyperplasia) and Marsh type III destructive (including intra-epithelial lymphocytosis, crypt hyperplasia and villous atrophy: partial, subtotal and total villous atrophy - Marsh type IIIA, Marsh type IIIB and Marsh type IIIC respectively). In Marsh type IIIA, partial villous atrophy, the villi were blunt and shortened. According to another publication, the pathologist scored samples as Marsh type IIIA if the villous/crypt ratio was less than $1: 1$ [8]. In Marsh type IIIB, subtotal villous atrophy, villi were clearly atrophic but still distinguished, and in Marsh type IIIC, total villous atrophy, villi were rudimentary with flat mucosa. The end point of follow-up in this study was recovery of intestinal mucosal alterations, with normalization of villous architecture by regression to Marsh type 0 or Marsh type I.

The initial group of 105 children was divided into two subgroups according to the degree of villous alterations described at diagnosis. The first subgroup consisted of 19 children with digestive symptoms and/or extra-intestinal manifestations

Table I. Cumulative rates of celiac children with villous atrophy achieving histologic recovery within $1-2$ years, 2 to 3 years, and after more than 3 years of gluten-free diet

\begin{tabular}{|lc|}
\hline $\begin{array}{l}\text { Surveillance time } \\
\text { [years] }\end{array}$ & $\begin{array}{r}\text { Proportion of children achieving } \\
\text { histologic remission, } n \text { (\%) }\end{array}$ \\
\hline $1-2$ & $70(81.4)$ \\
\hline $2-3$ & $79(91.8)$ \\
\hline$\geq 3$ & $84(97.6)$ \\
\hline
\end{tabular}

and positive celiac serology, having lymphocytic enteritis with crypt hyperplasia (Marsh type II lesions) at diagnosis. The second subgroup consisted of 86 CD children with villous atrophy (Marsh type III lesions) described at diagnosis.

\section{Statistical analysis}

The statistical analyis was performed using SPSS Statistics for Windows, version 17 (SPSS Inc., Chicago, Ill., USA). Means and standard deviations (SD) were calculated for numerical variables with a normal distribution, whereas for variables with a non-normal distribution, medians and ranges were calculated. The $\chi^{2}$ test was used to compare proportions expressed as percentages. The 95\% confidence interval $(\mathrm{Cl})$ was calculated for each predictive test. For all statistical analyses, a twotailed $p$-value $<0.05$ was considered significant.

\section{Results}

The global cumulative analysis of all 105 celiac children enrolled in the study indicated that 89/105 (84.7\%) children achieved histologic remission within 1 to 2 years after gluten withdrawal. After 2 to 3 years, histologic remission was achieved and maintained in 98/105 (93.3\%) cases, and after more than 3 years of gluten-free diet, the histologic restoration rate increased to $98 \%$ (103 of 105 cases).

The authors performed further detailed analysis of the two subgroups of children enrolled.

DQ2 and DQ8 haplotypes were analyzed to strengthen the CD diagnosis in all children without villous atrophy enrolled in the first subgroup. Fifteen of 19 patients with Marsh type II presented celiac-related DQ2 HLA and 4 children presented DQ8 HLA, supporting the diagnosis as celiac patients. Follow-up clinical, serological and histological parameters were monitored. None of these patients had associated selective IgA deficiency. In all these patients intestinal histologic alterations recovered to normal architecture (Marsh type 0) after 1 year of strict diet. Symptoms and laboratory parameters improved in all 19 children from the first month of gluten-free diet and serum IgA tTG antibodies normalized after a mean period of 11 weeks of diet ( \pm 2 weeks).

The overall histologic recovery timing profile in the second subgroup with villous atrophy is shown in Table I.

$97.6 \%$ of children with initial Marsh type III intestinal injuries achieved histologic remission after 3 years of gluten-free diet, with the majority recovering after the first year of diet.

The authors performed a further analysis in the second subgroup of $86 \mathrm{CD}$ children according to the severity of villous atrophy (Marsh type IIIA, type IIIB and type IIIC lesions) revealed at diagno- 
sis. There were 27 children (31.4\%) with partial villous atrophy (Marsh type IIIA), 34 (39.5\%) children with subtotal villous atrophy (Marsh type IIIB) and $25(29 \%)$ cases with total villous atrophy (Marsh type IIIC). The histologic remission profiles for children with villous atrophy at the initial diagnostic biopsy are depicted in Table II.

The majority of celiac children with villous atrophy recovered after 1 year of gluten-free diet. Children with initial intestinal lesions classified as Marsh type IIIA completely recovered within 2 to 3 years after gluten withdrawal, while all children with subtotal villous atrophy (Marsh type IIIB) achieved histologic remission after 3 years of gluten-free diet.

There was a statistically significant difference between the proportions of children with Marsh type IIIA, type IIIB and Marsh type IIIC who achieved histologic recovery within 1 to 2 years after gluten withdrawal. There were more children with partial (92.6\%) and subtotal villous atrophy (88.2\%) showing histologic improvement, compared to only $60 \%$ of patients with total villous atrophy who recovered within the first 2 years of diet, $p=0.01 ; 95 \% \mathrm{Cl}: 0.07-0.58$ and $p=0.02$ respectively, $95 \% \mathrm{Cl}$ : 0.02-0.53.

Children with total villous atrophy did not recover completely. Only 23 (92\%) achieved histologic remission after 3 years of follow-up. Two patients with persisting villous atrophy and concomitant selective IgA deficiency had symptoms of malabsorption and lower but still detectable IgG anti-tTG antibodies after more than 3 years of follow-up, despite a strict gluten-free diet. IgG anti-endomysial antibodies were negative in both cases. The intestinal lesions slightly regressed from total to partial villous atrophy after 3 years of follow-up.

The end stage of histologic improvement achieved by children with initial villous atrophy following a gluten-free diet on long-term follow-up is depicted in Table III.

Most of the cases with initial villous atrophy recovered to normal intestinal histology (Marsh type 0) on long-term follow-up. Globally, the improvement to Marsh type 0 was achieved in $71 \%$ of cases (61 from 86 children) on a gluten-free diet. In $26.7 \%$ of cases ( 23 from 86 children), the end stage histologic recovery was Marsh type I score after gluten withdrawal. In 2 cases with total villous atrophy (Marsh type IIIC) at diagnosis, histologic remission was not achieved. These two patients represent $8 \%$ of the Marsh type IIIC group and $2.3 \%$ reported to the entire cohort of 86 children with villous atrophy (Marsh type III lesions). They presented a slow morphologic regression from total to partial villous atrophy (Marsh type IIIA) after more than 3 years of gluten-free diet, but they did not recover to Marsh type 0 or type I, the stated end point of follow-up in this study.

There was no significant difference between the proportion of children with initial Marsh type IIIA (77.7\%) and Marsh type IIIB (76.4\%) lesions that recovered to normal intestinal histology (Marsh type 0), $p=1 ; 95 \% \mathrm{Cl}:-0.21-0.23$. Data analysis indicated higher proportions of children with Marsh type IIIA (77.7\%) and Marsh type IIIB (76.4\%) who achieved complete remission to normal intestinal histology (Marsh type 0), compared to Marsh type IIIC subgroup (56\%), although it did not reach statistical significance, $p=0.16$; $95 \% \mathrm{Cl}:-0.07-0.50$ and $p=0.16$ respectively, $95 \% \mathrm{Cl}:-0.07-0.48$.

\section{Discussion}

The results of this long-term follow-up study of children with CD showed that the histologic recovery after starting a gluten-free diet takes time (at least 1 year in $81.4 \%$ of children with different

Table II. Cumulative rates of histologic recovery among celiac children on gluten-free diet with different initial degrees of villous atrophy in long-term follow-up ( $\geq 3$ years)

\begin{tabular}{|lccc|}
\hline \multirow{2}{*}{$\begin{array}{l}\text { Surveillance time } \\
\text { [years] }\end{array}$} & \multicolumn{3}{c|}{ Rate of histologic recovery, $n$ (\%) } \\
\cline { 2 - 4 } & Marsh type IIIA (27) & Marsh type IIIB (34) & Marsh type IIIC (25) \\
\hline $1-2$ & $25(92.6)$ & $30(88.2)$ & $15(60)$ \\
\hline $2-3$ & $27(100)$ & $31(91.1)$ & $21(84)$ \\
\hline$\geq 3$ & $27(100)$ & $34(100)$ & $23(92)$ \\
\hline
\end{tabular}

Table III. End stage histologic score achieved by children in remission in the subgroup with villous atrophy

\begin{tabular}{|lccc|}
\hline Histologic recovery & \multicolumn{3}{c|}{ Histologic score at diagnosis, $n$ (\%) } \\
\cline { 2 - 4 } stage & Marsh type IIIA (27) & Marsh type IIIB (34) & Marsh type IIIC (25) \\
\hline Marsh type 0 & $21(77.7)$ & $26(76.4)$ & $14(56)$ \\
\hline Marsh type I & $6(22.2)$ & $8(23.5)$ & $9(36)$ \\
\hline Not recovered & $0(0)$ & $0(0)$ & $2(8)$ \\
\hline
\end{tabular}


degrees of villous atrophy) and is incomplete only in a small proportion of children (2.3\% persistent partial villous atrophy in long-term follow-up). The analysis of symptomatic celiac children with lymphocytic enteritis and crypt hyperplasia (Marsh type II lesions) showed a complete remission (100\%) to normal architecture (Marsh type 0) after 1 year of strict diet. The severity of intestinal alterations (according to Marsh score modified by Oberhuber) correlates with the histologic improvement profile and timing. The less severe intestinal lesions were accompanied by faster and more complete histologic recovery.

In children with non-responsive CD first of all strict gluten exclusion should be assessed. No overt sources of gluten intake were identified in our 2 patients with persistent villous atrophy. The 2 children who did not achieve histologic remission in the first 3 years were diagnosed with $C D$ and IgA deficiency. Both patients had heterozygous DQ2 haplotype. It is known that in IgA-deficient patients, the IgG celiac serology may be persistently elevated, despite histologic recovery [13]. Specific antibody synthesis is dependent on $T$ helper lymphocytes, $T$ cell priming and cytokine profiles and $B$ cell responsiveness may present malfunctions in IgA deficiency [13]. Therefore, the slow remission of IgG antibodies may be part of the immunoregulatory defect characteristic for selective IgA deficiency $[13,14]$.

To achieve complete gluten avoidance and appropriate surveillance after more than 3 years of non-response to gluten withdrawal, we provided exclusive enteral nutrition for 3 months to those 2 patients. As reported by previous papers, patients with $C D$ not responding to a gluten-free diet may benefit from additional dietary restrictions, such as an enteral elemental diet or total parenteral nutrition $[15,16]$. After this dietary intervention in both cases the serum level of IgG tTG antibody gradually decreased to normal and the digestive symptoms and malabsorption syndrome disappeared. The authors repeated the intestinal biopsies after 5 years of follow-up on a gluten-free diet and in both cases we found regression to Marsh type I and both patients remained asymptomatic.

Refractory celiac disease (RCD) should be suspected in $C D$ patients with persistent enteropathy in spite of good adherence to treatment with a gluten-free diet for at least 12 months $[17,18]$. The definition of RCD also includes the occurrence of severe clinical deterioration in diagnosed CD, which requires rapid intervention independent of the duration of the gluten-free diet [19]. Two types of RCD have been recognized on the basis of immune phenotyping of intraepithelial lymphocytes: type I - without or with less than $20 \%$ aberrant CD3- CD4- T-cells; and type II - with over $20 \%$ aberrant T-cells in the intestinal mucosa [20]. The RCD has been observed almost only in adult-onset $C D$, while in children it is a rare condition [19]. Positive CD-specific serology can be present in $19-30 \%$ of patients with RCD despite good compliance with a gluten-free diet; thus, positive CD-specific serology does not necessarily exclude the diagnosis of RCD [21]. Although specific immunochemical testing for RCD was not available in our center, we ruled out RCD in these two cases due to clinical, serological and histological recovery after exclusive enteral nutrition. There was no need for corticosteroids or immunosuppressive drugs. Possible low-grade gluten contamination by hidden sources could have contributed to the initially non-responsive status of these 2 patients, inducing slow histological recovery.

In this study, remission of intestinal pathologic alterations, with normalization of villous architecture by regression to Marsh type 0 or Marsh type I, was considered the end point of follow-up. The authors did not perform further follow-up biopsies in children with minimal histologic injuries as infiltrative lymphocytic enteritis (Marsh type I) until Marsh type 0 would have been achieved, due to ethical reasons, as the symptoms had disappeared and serological tests normalized.

The ESPGHAN guide for CD management in children did not specifically define histologic remission. The guide recommended that patients should be followed up regularly for symptomatic improvement and normalization of CD specific antibody tests [3]. It is also stated that in patients fulfilling the diagnostic criteria for CD it is unnecessary to perform small-bowel biopsies on a gluten-free diet. Only if there is no clinical response to the gluten-free diet in symptomatic patients may the investigations include further biopsies [3].

Intestinal histologic recovery in celiac children has still not been extensively studied and there are no conclusions about persistence of crypt hyperplasia and intra-epithelial lymphocyte infiltration. It is not clear if reappearance of normal villi would be accompanied by disappearance of crypt hyperplasia and decrease of the intraepithelial lymphocyte density. This was the main reason behind the design of this study. We consider that further, larger studies on this topic should investigate the long-term consequences of persistent minimal intestinal alterations as risk for malignancy especially in pediatric onset CD.

The importance of correct orientation of the specimen has long been recognized. The cutting should be perpendicular to the mucosal surface in order to avoid false diagnoses [22]. Incorrect biopsy sample orientation will determine cross-sectioning of the crypts and loss of evidence of crypt hyperplasia, being one of the main reasons for misinterpreting CD histology $[23,24]$. 
The intestinal samples obtained by endoscopy biopsy forceps are often small, making the correct orientation difficult. In all biopsy samples in this study, the initial sectioning of the crypts was carefully assessed by the pathologist, in order to avoid the reading of tangential cuttings and false results. This observation was of great importance for an accurate interpretation of the histologic recovery stage.

In this study, all follow-up small intestinal biopsies were taken only from the second part of the duodenum. The bulb biopsies were avoided for monitoring histologic remission for several reasons. Until 2005, guidelines did not allow biopsy sampling from the bulb [25]. Even in 2011 the Italian pathologist consensus discouraged the use of bulb biopsy due to several potential sources of error [26]. Starting in 2012, the new ESPGHAN guidelines recommend obtaining also bulb biopsies for CD diagnosis [3]. These guidelines did not taken into account the particularities of the bulb samples. The most common interfering factors mentioned are lymphoid follicles, Brunner's glands, the potential damaging effects of nearby gastric acid and susceptibility to mucosal alterations caused by gastrointestinal infections [26]. All of these could cause shortened villi in the bulb, mimicking $C D$, while the distal duodenum remained normal [26, 27].

There are only a few pediatric studies in the literature debating the subject of histologic remission among celiac children. Our results are similar to other published studies regarding intestinal histological restoration in pediatric patients. Grefte et al. [28] reported slow and incomplete histologic and functional recovery in 22 adults with gluten enteropathy after 24 to 48 months of gluten exclusion. Studying a mixed cohort of 158 celiac children and adult patients Wahab et al. [8] reported that only $65.0 \%$ of the patients achieved histologic remission within 2 years. Moreover, within 5 years, $85.3 \%$ were in remission, and in longterm follow-up $89.9 \%$ of patients showed normalization of villous architecture. Wahab showed that in a subgroup of 25 children included in the total group of 158 patients, 24 (96\%) achieved histologic restoration within 2 years and $100 \%$ recovered in long-term follow-up [8].

The histologic recovery seems to be faster in children than in adult patients and it has a propensity to be more complete in pediatric patients.

A recently described alternative to esophago-gastro-duodenoscopy to examine the entire small bowel is capsule endoscopy. According to Krok et al. [29] the patients' preference between standard upper gastrointestinal endoscopy and capsule endoscopy is in favor of the capsule. The patients with chronic gastro-intestinal conditions would prefer a surveillance program that used capsule endoscopy over upper gastrointestinal endoscopy [29]. But this endoscopic method is not able to provide biopsy samples, so it could not be indicated for intestinal recovery assessment in gluten enteropathy. Gastrointestinal endoscopy is a minimally invasive and highly effective method [30], available for all age groups, capable of sampling intestinal tissue. Therefore it is the main tool indicated for assessment of histologic recovery in CD patients.

In conclusion, histologic recovery in $C D$ after starting a gluten-free diet in children takes at least 1 year and might be incomplete only in a small proportion of children, mainly with IgA immunodeficiency. There is no need for routine histologic assessment in all celiac children after gluten withdrawal. Considering the risk for secondary complications such as osteopenia and malignancies (enteropathy associated T-cell lymphoma), this study underlines the importance of systematic follow-up including duodenal biopsies among CD children with persistent malabsorption syndrome despite a strict gluten-free diet.

\section{Conflict of interest}

The authors declare no conflict of interest.

\section{References}

1. Chou R, Bougatsos C, Blazina I, Mackey K, Grusing S, Selph S. Screening for celiac dsease: evidence report and systematic review for the US Preventive Services Task Force. JAMA 2017; 317: 1258-68.

2. Kelly CP, Bai JC, Liu E, Leffler DA. Advances in diagnosis and management of celiac disease. Gastroenterology 2015; 148: 1175-86.

3. Husby S, Koletzko S, Korponay-Szabó IR, et al. European Society for Pediatric Gastroenterology, Hepatology and Nutrition Guidelines for the Diagnosis of Coeliac Disease. J Pediatr Gastroent Nutr 2012; 54: 136-60.

4. Marsh MN. Gluten, major histocompatibility complex and the small intestine: a molecular and immunobiologic approach to the spectrum of gluten sensitivity ("celiac sprue"). Gastroenterology 1992; 102: 330-54.

5. Wahab PJ, Crusius JBA, Meijer JWR, Mulder CJ. Gluten challenge in borderline gluten sensitive enteropathy. Am J Gastroenterol 2001; 96: 1464-9.

6. Kurppa K, Collin P, Viljamaa M, et al. Diagnosing mild enteropathy celiac disease: a randomized, controlled clinical study. Gastroenterology 2009; 136: 816-23.

7. Taavela J, Koskinen $\mathrm{O}$, Huhtala $\mathrm{H}$, et al. Validation of morphometric analyses of small-intestinal biopsy readouts in celiac disease. PLoS One 2013; 8: e76163.

8. Wahab P, Meijer J, Mulder C. Histologic follow-up of people with celiac disease on a gluten-free diet. Am J Clin Pathol 2002; 118: 459-63.

9. Oberhuber G. Histopathology of celiac disease. Biomed Pharmacother 2000; 54: 368-72.

10. Corazza GR, Villanacci V. Coeliac disease. J Clin Pathol 2005; 58: 573-4.

11. Rubio-Tapia A, Rahim MW, See JA, Lahr BD, Wu TT, Murray JA. Mucosal recovery and mortality in adults with 
celiac disease after treatment with a gluten free diet. Am J Gastroenterol 2010; 105: 1412-20.

12. Pallav K, Xu H, Leffler DA, Kabbani T, Kelly CP. Immunoglobulin A deficiency in celiac disease in the United States. J Gastroenterol Hepatol 2016; 31: 133-7.

13. Chow MA, Lebwohl B, Reilly NR, Green PH. Immunoglobulin A deficiency in celiac disease. J Clin Gastroenterol 2012; 46: 850-4.

14. Korponay-Szabo IR, Dahlbom I, Laurila K, et al. Elevation of IgG antibodies against tissue transglutaminase as a diagnostic tool for coeliac disease in selective IgA deficiency. Gut 2003; 52: 1567-71.

15. Olaussen RW, Lovik A, Tollefsen S, et al. Effect of elemental diet on mucosal immunopathology and clinical symptoms in type 1 refractory celiac disease. Clin Gastroenterol Hepatol 2005; 3: 875-85.

16. Mandal A, Mayberry J. Elemental diet in the treatment of refractory coeliac disease. Eur J Gastroenterol Hepatol 2001; 13: 79-80.

17. Biagi F, Corazza GR. Defining gluten refractory enteropathy. Eur J Gastroenterol Hepatol 2001; 13: 561-5.

18. Mooney PD, Evans KE, Singh S, Sanders DS. Treatment failure in coeliac disease: a practical guide to investigation and treatment of non-responsive and refractory coeliac disease. J Gastrointest Liver Dis 2012; 21: 197-203.

19. Jańczyk W, De Roo JHC, Schweizer J, Socha J, Socha P, Mearin L. Coeliac disease not responding to a gluten free diet in children: case studies and literature review. Dev Period Med 2015; 29: 162-6.

20. Cellier C, Patey N, Mauvieux L, et al. Abnormal intestinal intraepithelial lymphocytes in refractory sprue. Gastroenterology 1998; 114: 471-81.

21. Rubio-Tapia A, Kelly DG, Lahr BD, Dogan A, Wu TT, Murray JA. Clinical staging and survival in refractory celiac disease: a single center experience. Gastroenterology 2009; 136: 99-107.

22. Ravelli A, Villanacci V. Tricks of the trade: how to avoid histological pitfalls in celiac disease. Pathol Res Pract 2012; 208: 197-202.

23. Arguelles-Grande C, Tennyson CA, Lewis SK, Green PH, Bhagat G. Variability in small bowel histopathology reporting between different pathology practice settings: impact on the diagnosis of coeliac disease. J Clin Pathol 2012; 65: 242-7.

24. Rostom A, Murray JA, Kagnoff MF. American Gastroenterological Association (AGA) institute technical review on the diagnosis and management of celiac disease. Gastroenterology 2006; 131: 1981-2002.

25. Hill ID, Dirks MH, Liptak GS, et al. North American Society for Pediatric Gastroenterology, Hepatology and Nutrition Clinical Guideline. Guideline for the diagnosis and treatment of celiac disease in children: recommendations of the North American Society for Pediatric Gastroenterology, Hepatology and Nutrition. J Pediatr Gastroenterol Nutr 2005; 40: 1-19.

26. Villanacci V, Ceppa P, Tavani E, Vindigni C, Volta U, Gruppo Italiano Patologi Apparato Digerente (GIPAD), Società Italiana di Anatomia Patologica e Citopatologia Diagnostica/International Academy of Pathology, Italian division (SIAPEC/IAP). Coeliac disease: the histology report. Dig Liver Dis 2011; 43 (Suppl 4): S385-95.

27. Chang F, Mahadeva U, Deere H. Pathological and clinical significance of increased intraepithelial lymphocytes (IELs) in small bowel mucosa. APMIS 2005; 113: 385-99.

28. Grefte JM, Bouman JG, Grond J, Jansen W, Kleibeuker JH. Slow and incomplete histological and functional recovery in adult gluten sensitive enteropathy. J Clin Pathol 1988; 41: 886-91.
29. Krok KL, Wagennar RR, Kantsevoy SV, Thuluvath PJ. Esophageal capsule endoscopy is not the optimal technique to determine the need for primary prophylaxis in patients with cirrhosis. Arch Med Sci 2016; 12: 365-71.

30. Aday U, Duman M, Deđer KC, et al. Endoscopic approach to anastomotic leaks: partially covered self-expandable stents. A single center's experience. Arch Med Sci Civil Dis 2017; 2: e29-e34. 